\title{
Heuschnupfentherapie auf Kosten der Patienten
}

\author{
Die OTC-Ausnahmeliste sowie diverse KV-Rundschreiben haben bei \\ Ärzten Verwirrung darüber gestiftet, welche Präparate sie noch zu \\ Lasten der gesetzlichen Krankenversicherung verordnen können. \\ Doch auch bei den Patienten ist die Verunsicherung groß.
}

S eit dem 1. Januar 2004 können rezeptfreie Antihistaminika nur noch bei Kindern bis zum 12. Lebensjahr und bei Jugendlichen mit Entwicklungsstörungen bis zum 18. Lebensjahr zu Lasten der gesetzlichen Krankenversicherung (GKV) verordnet werden. Ausnahmen hat der Gemeinsame Bundesausschuss in der seit dem 1. April 2004 geltenden OTC-Ausnahmeliste festgelegt:

- Nur in Notfallsets zur Behandlung einer lnsektengiftallergie,

— zur Behandlung schwerer, rezidivierender Urtikaria und

- bei schwerwiegendem, anhaltenden Pruritus

werden nicht verschreibungspflichtige Antihistaminika noch erstattet.

Prof. Dr. Thomas Fuchs, Präsident des Ärzteverbandes Deutscher Allergologen (ÄDA), ist mit der Ausnahmeliste nicht einverstanden: „Es ist unverständlich, dass rezeptfreie Antihistaminika nicht auch in Notfallsets für Naturlatexund Nahrungsmittelallergiker sowie bei der Therapie eines rezidivierenden Angioödems und der schweren, saisonalen Rhinokonjunktivitis mit monatelangen Beschwerden erstattet werden."

\section{Neuere Substanzen weiter erstattungsfähig}

Die Behandlung der allergischen Rhinitis bleibt also den Betroffenen weitgehend selbst überlassen und erfolgt ohne ärztliche Therapiekontrolle. Zusätzlich hängt sie stark vom Geldbeutel der Patienten ab. „Viele Allergiker nehmen ihre Antihistaminika trotz starker Beschwerden nur noch alle paar Tage, um Geld zu sparen!“, warnt Andrea Wallrafen vom Deutschen Allergie- und Asthmabund (DAAB). Betroffene hätten am DAAB Beratungstelefon außerdem versichert, dass viele Ärzte Medikamente gegen
Heuschnupfen nur noch auf Privatrezept oder gar nicht verordnen würden - und dies sogar in Fällen, in denen die Patienten in den Vorjahren noch rezeptpflichtige Antihistaminika erhalten hätten, die weiterhin erstattungsfähig sind.

Dabei ist die Verordnung der neueren, verschreibungspflichtigen Antihistaminika wie Desloratadin, Ebastin, Fexofenadin, Levocetirizin und Mizolastin bei allergischer Rhinitis unproblematisch. Sie sind bei Vorliegen der medizinischen Notwendigkeit und Zweckmäßigkeit nach $\$ 12$ SGB V unter Berücksichtigung des Wirtschaftlichkeitsgebotes erstattungsfähig. Die verschreibungspflichtigen Antihistaminika können zu Lasten der GKV verordnet werden, wenn der Patient bereits mit einem solchen Präparat eingestellt und gut therapiert ist, wenn die mit der Einnahme rezeptfreier Antihistaminika verbundenen Risiken wie kardiotoxische Nebenwirkungen, Sedierung, psychomotorische Störungen sowie Wechselwirkungen vermieden werden sollen oder wenn der Patient ein stärker wirksames Antihistaminikum benötigt, das insbesondere die nasale Obstruktion lindert. „Der behandelnde Arzt hat weiterhin die Therapiefreiheit, das für seinen Patienten am besten geeignete
Präparat auszuwählen“, erklärt Prof. Dr. Claus Bachert von der Deutschen Gesellschaft für Allergologie und klinische lmmunologie (DGAI).

\section{Heuschnupfen keine Bagatellerkrankung}

Bachert kritisiert die OTC-Ausnahmeregelung für Antihistaminika aus einem weiteren Grund: Die allergische Rhinitis wird offensichtlich als Bagatellerkrankung eingestuft. Bei Heuschnupfen drohen aber ohne adäquate Therapie eine fortschreitende Verschlechterung und Folgeerkrankungen. Nach neueren Untersuchungen leidet etwa ein Drittel der Patienten mit allergischer Rhinitis unter einer persistierenden Erkrankung. Die Beschwerden bestehen bei diesen $\mathrm{Pa}$ tienten fast täglich über einen Zeitraum von 4 Wochen, oft sogar über Monate. Es handelt sich somit um eine chronische Atemwegserkrankung mit erheblicher Komorbidität und weitreichenden Auswirkungen auf die Leistungsfähigkeit und Lebensqualität der Patienten. Die allergische Rhinitis erfordert eine konsequente, ärztlich kontrollierte Therapie, um den Übergang zu Asthma oder die Ausweitung zu einer chronischen Sinusitis zu verhindern. Auch Schlafstörungen müssen durch eine geeignete Medikation verhindert werden, damit bei den Patienten die Arbeitsfähigkeit erhalten bleibt. ÄDA-Präsident Fuchs bemängelt: „Es darf nicht sein, dass jetzt die Apothekenmitarbeiterin über die Therapie der allergischen Rhinitis mit bronchialer $\mathrm{Hy}$ perreaktivität entscheidet. Damit werden unsere Bemühungen konterkariert, die Qualität der allergologischen Versorgung im Sinne einer evidenzbasierten Medizin zu verbessern!"

$\mathrm{mm}$

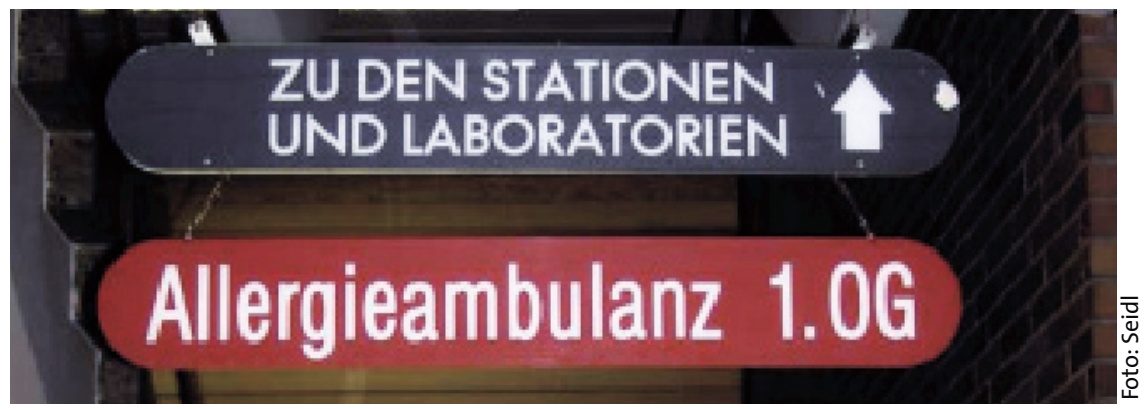

Immer mehr Patienten mit allergischer Rhinitis suchen erst gar nicht mehr den Arzt auf, weil sie glauben, die Therapie ohnehin selbst zahlen zu müssen. 